\title{
CONSTRUCTION OF THE NUMERICAL AND SEMI-ANALYTICAL SOLUTIONS OF THE RIGID EARTH ROTATION AT A LONG TIME INTERVALS
}

\author{
V.V. Pashkevich \\ Central (Pulkovo) Astronomical Observatory of RAS \\ Pulkovskoe shosse, 65/1, 196140, St.Petersburg, Russia \\ e-mail: pashvladvit@yandex.ru
}

\begin{abstract}
This research is the continuation of our studies of the rigid Earth rotation at a long time intervals (Pashkevich V.V. and Eroshkin G.I., 2005). The main purpose of this investigation is the construction of the new high-precision Rigid Earth Rotation Series 2012 (RERS2012), dynamically adequate to the JPL DE406/LE406 ephemeris (Standish E. M., 1998). The dynamics of the rotational motion of the rigid Earth is studied numerically by using Rodrigues-Hamilton parameters over 2000 and 6000 years. The numerical solution of the rigid Earth rotation is implemented with the quadruple precision of the calculations. The orbital motions of the disturbing celestial bodies are defined by the DE406/LE406 ephemeris. The initial conditions of the numerical integration are taken from SMART97 (Bretagnon P. et al., 1998) and S9000 (Pashkevich V.V. and Eroshkin G.I. 2005). The results of the numerical solutions of the problem are compared with the semi-analytical solutions of the rigid Earth rotation (SMART97 and S9000, respectively) with respect to the fixed ecliptic of epoch J2000. The investigation of these discrepancies is carried out by the least squares and spectral analysis methods for the relativistic (Kinematical) case, in which the geodetic perturbations (the most essential relativistic perturbations) in the Earth rotation are taken into account. As a result, the Rigid Earth Rotation Series (RERS2012) is constructed, which is dynamically adequate to the DE406/LE406 ephemeris over 2000 and 6000 years. The discrepancies between the new numerical solutions and the semi-analytical solutions of MRS2012 do not surpass $12 \mu$ as over 2000 year time interval and 2 mas over 6000 year time interval. Thus, the result of the comparison demonstrates a good consistency of RERS2012 series with the DE406/LE406 ephemeris.
\end{abstract}

Keywords: the rotation of the rigid Earth, ephemeris, least-squares method, spectral analysis method.

\section{INTRODUCTION}

In the previous research (Pashkevich V.V. and Eroshkin G.I. 2005) the high-precision rigid Earth rotation semi-analytical solutions of S9000, dynamically adequate to the DE404/LE404 ephemeris, was constructed over 2000 year time interval.

The main purpose of this investigation is the construction of the new high-precision rigid Earth rotation series (RERS2012A/B and RERS2012C/D), dynamically adequate to the JPL DE406/LE406 ephemeris (Standish E. M., 1998), over 2000 and 6000 years (full time interval of the DE406/LE406 ephemeris existence), respectively. The initial epoch of the 
numerical integration is 1 January $2000(\mathrm{JD}=2451545.0)$. The initial conditions of the numerical integration are taken from SMART97 (Bretagnon P. et al., 1998) for the construction of the series RERS2012A/C and from S9000 (Pashkevich V.V. and Eroshkin G.I. 2005) for the construction of the series RERS2012B/D. The mathematical model of the present investigation is identical to that used by Pashkevich and Eroshkin (2005).

\section{MATHEMATICAL MODEL OF THE PROBLEM}

The numerical solution of the problem is obtained by solving the Lagrange differential equations of the second kind for the rigid Earth rotation with respect to the fixed ecliptic and equinox of epoch $\mathrm{J} 2000$.

The Lagrange differential equations of the second kind

$$
\frac{d}{d t} \frac{\partial L}{\partial \dot{\lambda}_{i}}-\frac{\partial L}{\partial \lambda_{i}}=0, \quad i=0,1,2,3,
$$

are solved, where $L=T+U, U$ is the force function of the gravitational interaction of the Earth with the disturbing bodies (the Moon, the Sun and the major planets). The force function $U$ is expanded in the spherical harmonics and only the terms with the coefficients $\mathrm{C}_{\mathrm{j} 0}$ for $\mathrm{j}=2, \ldots, 5, \mathrm{C}_{22}, \mathrm{C}_{3 \mathrm{k}}, \mathrm{S}_{3 \mathrm{k}}$ for $\mathrm{k}=1,2,3, \mathrm{C}_{41}, \mathrm{~S}_{41}$ are used (as in the models for the construction SMART97 and S9000). The orbital motions of the disturbing bodies are defined by the DE406/LE406 ephemeris. The kinetic energy of the rotational motion of the rigid Earth, which takes into account the essential part of the geodetic rotation (Eroshkin G.I. et al., 2002), is expressed as

$$
T=\frac{1}{2}\left(A \omega_{* 1}^{2}+B \omega_{* 2}^{2}+C \omega_{* 3}^{2}\right),
$$

where $\bar{\omega}_{*}=\bar{\omega}+\bar{\sigma} \quad$ is vector of the Earth angular rotation including the geodetic rotation and $\bar{\omega}$ is Newtonian vector of the Earth angular rotation.

The vector of the angular velocity of the geodetic rotation of the Earth, which is the most essential relativistic component of the Earth rotational motion around the proper center of mass, has the following expression:

$$
\bar{\sigma}=\sum_{j \neq \oplus} \frac{G m_{j}}{c^{2}\left|\bar{R}_{j}-\bar{R}_{\oplus}\right|^{3}}\left\{\left(2 \dot{\bar{R}}_{j}-\frac{3}{2} \dot{\bar{R}}_{\oplus}\right) \times\left(\bar{R}_{j}-\bar{R}_{\oplus}\right)\right\} .
$$

Here $c$ is the velocity of light; $G$ is the gravitational constant; $m_{j}$ is the mass of a disturbing body $j ; \quad \bar{R}_{\oplus}, \dot{\bar{R}}_{\oplus}, \bar{R}_{j}, \dot{\bar{R}}_{j}$ are the vectors of the barycentric position and velocity of the Earth and body $j$. The symbol $\times$ means a vector product; the subscript $\oplus$ correspond to the Earth and the subscript $j$ correspond to the Moon, the major planets and the Sun. 
The Rodrigues - Hamilton parameters

$\lambda_{0}=\cos \frac{\theta}{2} \cos \frac{\psi+\varphi}{2}, \lambda_{1}=\sin \frac{\theta}{2} \cos \frac{\psi-\varphi}{2}, \lambda_{2}=\sin \frac{\theta}{2} \sin \frac{\psi-\varphi}{2}, \lambda_{3}=\cos \frac{\theta}{2} \sin \frac{\psi+\varphi}{2}$

are the functions of the Euler angles $\psi, \theta$ and $\varphi$. These parameters are bounded variables. It is very important for the numerical solution of the problem.

The expressions for the discrepancies between the numerical solution of the rigid Earth rotation and the semi-analytical solutions (SMART97 and S9000, respectively) are

$$
\left.\begin{array}{l}
\Delta \psi=\sum_{k=0}^{6} \psi_{k} t^{k}+\sum_{j} \sum_{k=0}^{4}\left[\psi_{S j k} \sin \left(v_{j 0}+v_{j 1} t\right)+\psi_{C j k} \cos \left(v_{j 0}+v_{j 1} t\right)\right] t^{k} \\
\Delta \theta=\sum_{k=0}^{6} \theta_{k} t^{k}+\sum_{j} \sum_{k=0}^{4}\left[\theta_{S j k} \sin \left(v_{j 0}+v_{j 1} t\right)+\theta_{C j k} \cos \left(v_{j 0}+v_{j 1} t\right)\right] t^{k} \\
\Delta \varphi=\sum_{k=0}^{6} \varphi_{k} t^{k}+\sum_{j} \sum_{k=0}^{4}\left[\varphi_{S j k} \sin \left(v_{j 0}+v_{j 1} t\right)+\varphi_{C j k} \cos \left(v_{j 0}+v_{j 1} t\right)\right] t^{k}
\end{array}\right\}
$$

where $\psi$ is the longitude of the ascending node of the Earth's dynamical equator on the fixed ecliptic J2000; $\theta$ is the angle of the inclination of the Earth's dynamical equator to the fixed ecliptic J2000; $\varphi$ is the proper rotation angle of the Earth between the ascending node of the Earth's dynamical equator and the principal axis of the minimum moment of inertia; $v_{j 0}, v_{j 1}$ are the phases and the frequencies of the corresponding semi-analytical solutions, respectively; $j=1, \ldots, 4113 ; t$ is the time in the Julian days; $\psi_{k}, \theta_{k}, \varphi_{k}$ are the coefficients of the secular terms; $\psi_{s j k}, \theta_{s j k}, \varphi_{s j k}, \psi_{c j k}, \theta_{c j k}, \varphi_{c j k}$ are the coefficients of the periodic and Poisson terms.

The high-precision numerical integration method HIPPI (Eroshkin G.I., 2000) was applied.

\section{ITERATIVE ALGORITHM}

The result of the comparison of the numerical solutions and semi-analytical solutions is studied by means the iterative algorithm:

1. The numerical solution of the rigid Earth rotation is implemented with the quadruple precision of the calculations. The initial conditions are computed by the semianalytical solution of the rigid Earth rotation (SMART97 or S9000). The discrepancies between the numerical solution and the semi-analytical solution are obtained in Euler angles over all the investigation time intervals with one day spacing.

2. The investigation of the discrepancies is carried out by the least squares method (LSQ) and by the spectral analysis (SA) method (Pashkevich V.V. and Eroshkin G.I., 2005). The secular terms are defined by LSQ. The set of the frequencies of the semianalytical solution is used without a change. Only the coefficients of the periodical terms and the coefficients of the Poisson terms are improved. The secular, periodic and Poisson terms representing the new high-precision rigid Earth rotation series RERS2012Xi (where $\mathrm{i}$ is a number of the iteration, $\mathrm{X}=\mathrm{A}, \mathrm{B}, \mathrm{C}, \mathrm{D}$ is the index of the series) are determined: 


$$
\left.\begin{array}{l}
\psi_{\text {RERS2012Xi }}=\Delta \psi_{\mathrm{i}-1}+\psi_{\text {RERS2012Xi-1 }} \\
\theta_{\text {RERS2012Xi }}=\Delta \theta_{\mathrm{i}-1}+\theta_{\text {RERS2012Xi-1 }} \\
\varphi_{\text {RERS2012Xi }}=\Delta \varphi_{\mathrm{i}-1}+\varphi_{\text {RERS2012Xi-1 }}
\end{array}\right\} .
$$

The series with index A and C are constructed from series SMART97 over 2000 and 6000 years, respectively. The series with index B and D are constructed from series S9000 over 2000 and 6000 years, respectively.

3. The numerical solution of the rigid Earth rotation is constructed anew with the new initial conditions, which are calculated by RERS2012Xi.

4. Steps 2 and 3 are repeated till the best results for the discrepancies between new numerical solution and new RERS2012Xi are obtained.

\section{RESULTS}

The previous investigation of the rigid Earth rotation over 2000 year time interval (Pashkevich and Eroshkin, 2005) was carried out for DE404/LE404 ephemeris.

In Figure 1 the discrepancies are depicted between the numerical and SMART97 semianalytical solutions of the Earth rotation after formal removal of the secular trends.

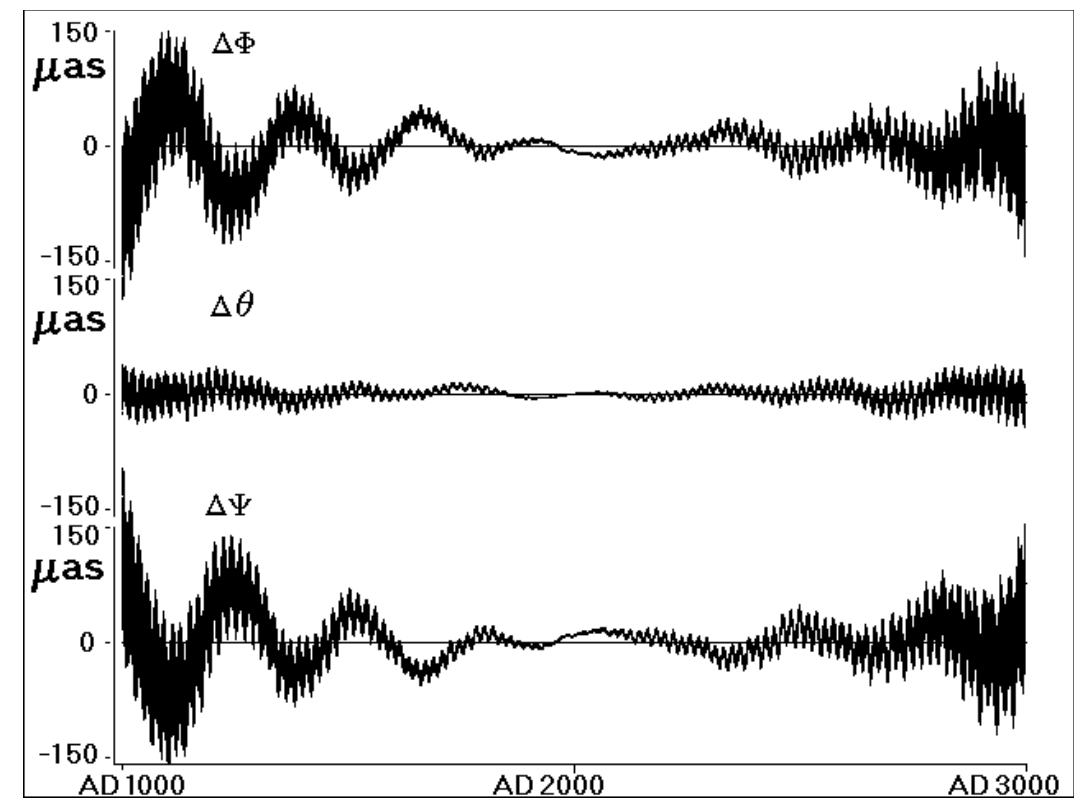

Fig. 1. Discrepancies between the numerical and SMART97 semi-analytical solutions of the Earth rotation (for the DE404/LE404 ephemeris) over 2000 years after formal removal of the secular trends (Pashkevich V.V. and Eroshkin G.I., 2005)

In Figure 2 the discrepancies are depicted between the numerical and S9000 semi-analytical solutions of the Earth rotation after formal removal of the secular trend in the proper rotation angle.

At first this investigation is carried out on 2000 years time interval for DE406/LE406 ephemeris. The discrepancies between the numerical and SMART97 semi-analytical solutions of the rigid Earth rotation (for the DE406/LE406 ephemeris) are obtained after formal removal of the secular trends in the Euler angles over 2000 year time interval with one day spacing (presented in Figure 3). 
The residuals in Figure 1 and Figure 3 are similar to each other and do not exceed $150 \mu$ as.

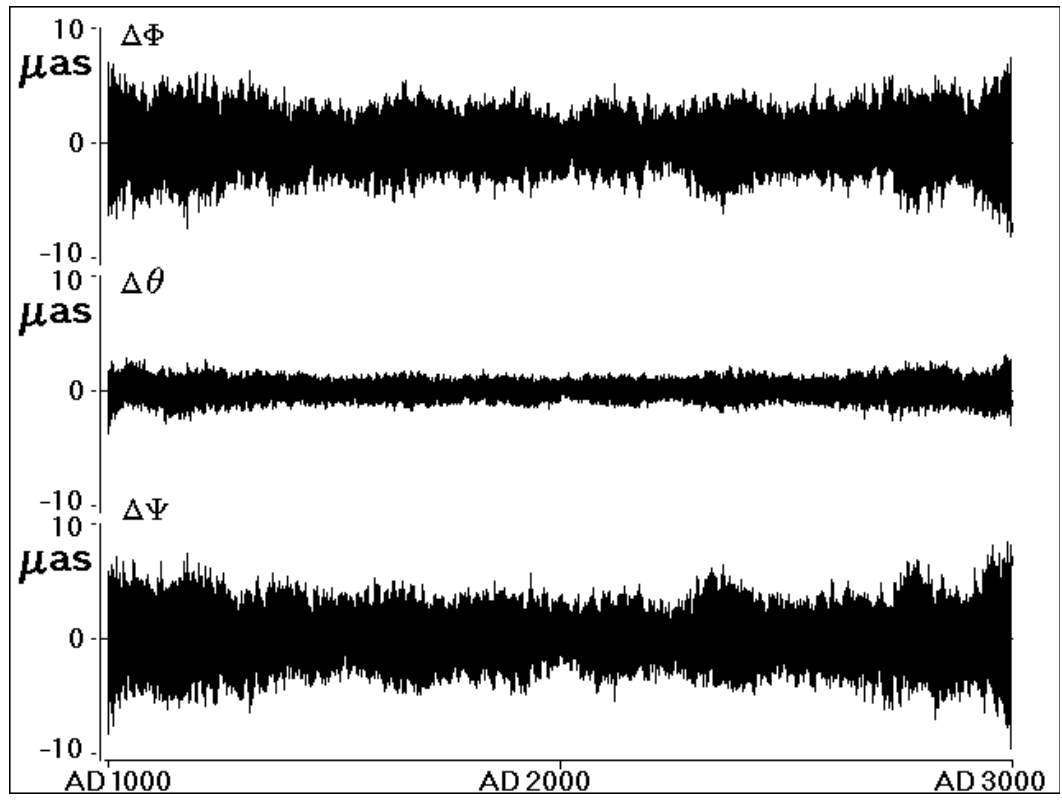

Fig. 2. Discrepancies between the numerical and S9000 semi-analytical solutions of the Earth rotation (for the DE404/LE404 ephemeris) over 2000 years after formal removal of the secular trend in the proper rotation angle (Pashkevich V.V. and Eroshkin G.I., 2005)

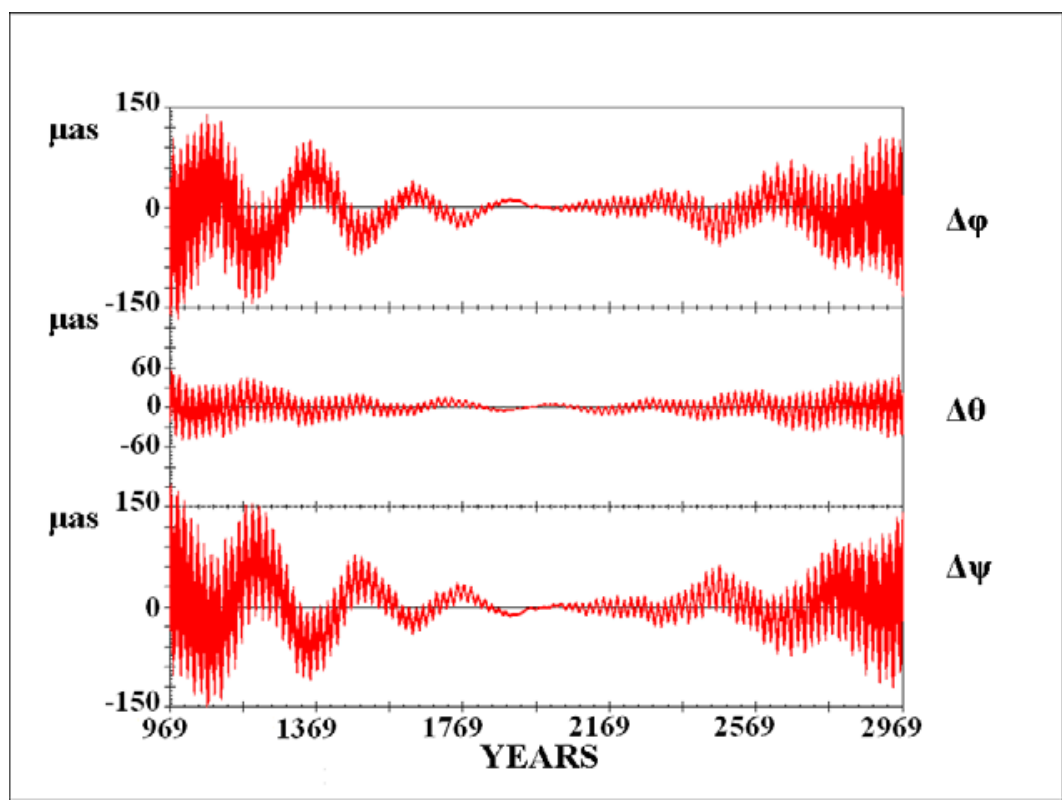

Fig. 3. Discrepancies between the numerical and SMART97 semi-analytical solutions of the Earth rotation (for the DE406/LE406 ephemeris) over 2000 years after formal removal of the secular trends

The appearance of the periodic and Poisson residuals (presented in Figure 3) is due to the lack of precision of the periodic and Poisson terms in the semi-analytical solution SMART97.

In Figure 4 the discrepancies are depicted between the numerical and RERS2012A-1 semi-analytical solutions of the Earth rotation over 2000 years after formal removal of the secular trends. The process of the iterative algorithm is finished after first iteration as in the previous investigation, where as the result was constructed semi-analytical solution S9000. The residuals in Figure 2 and Figure 4 do not exceed $10 \mu$ as and $12 \mu$ as, respectively. The 
residuals after the first iteration (presented in Figure 4) are better than the discrepancies between the numerical and SMART97 semi-analytical solutions (presented in Figure 3). So, the RERS2012A-1 solution is more accurate than SMART97.

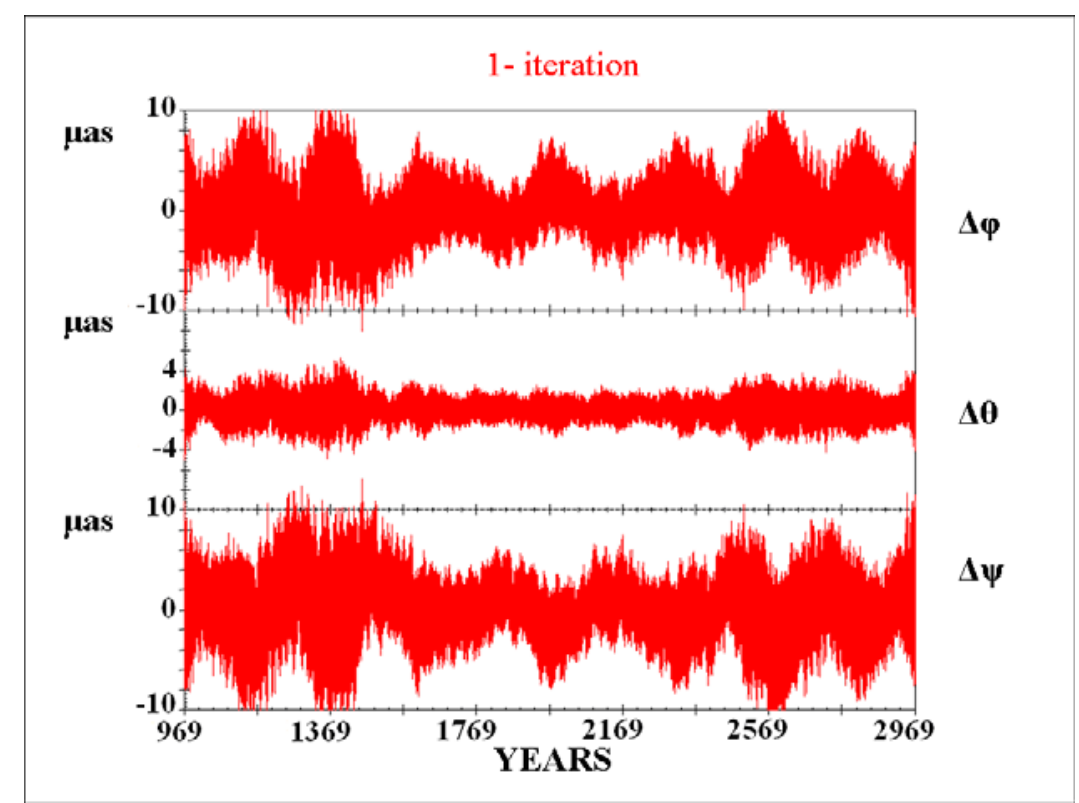

Fig. 4. Discrepancies between the numerical and RERS2012A-1 semi-analytical solutions of the Earth rotation (for the DE406/LE406 ephemeris) over 2000 years after formal removal of the secular trends

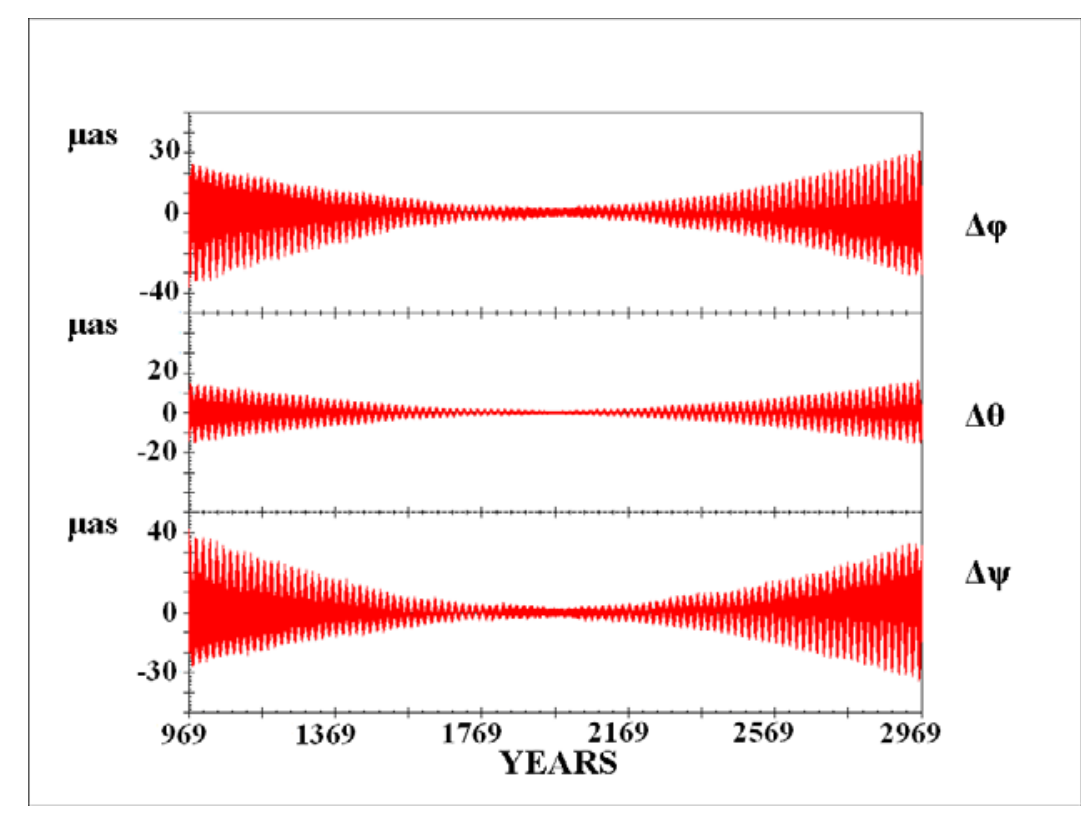

Fig. 5. Discrepancies between the numerical and S9000 semi-analytical solutions of the Earth rotation (for the DE406/LE406 ephemeris) over 2000 years after formal removal of the secular trends

The discrepancies between the numerical and S9000 semi-analytical solutions of the rigid Earth rotation (for the DE406/LE406 ephemeris) are obtained after formal removal of the secular trends in the Euler angles over 2000 year time interval with one day spacing (presented in Figure 5). Here, the residuals do not exceed $40 \mu$ as. So, the residuals between the numerical and S9000 semi-analytical solutions (presented in Figure 5) are better than the 
residuals between the numerical and SMART97 semi-analytical solutions (presented in Figure 3).

In Figure 6 the discrepancies are depicted between the numerical and RERS2012B-1 semi-analytical solutions of the Earth rotation over 2000 years after formal removal of the secular trend in the proper rotation angle. The residuals after the first iteration of the iterative algorithm do not exceed $10 \mu$ as.

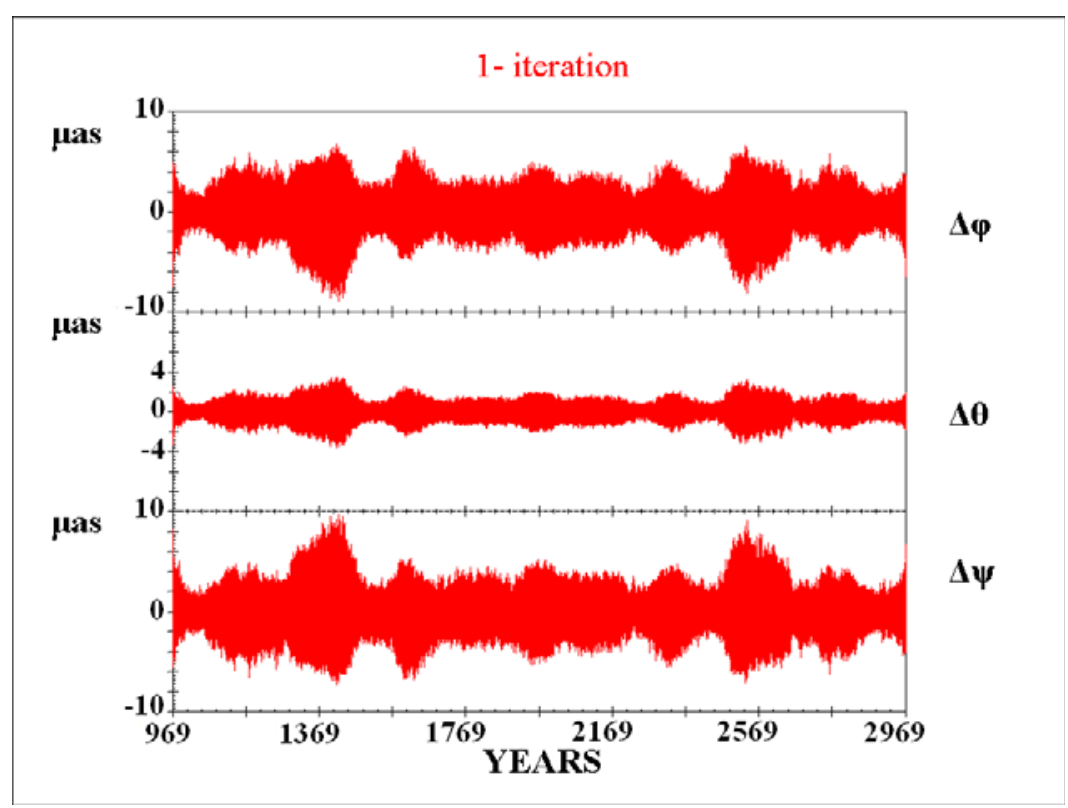

Fig. 6. Discrepancies between the numerical and RERS2012B-1 semi-analytical solutions of the Earth rotation (for the DE406/LE406 ephemeris) over 2000 years after formal removal of the secular trend in the proper rotation angle

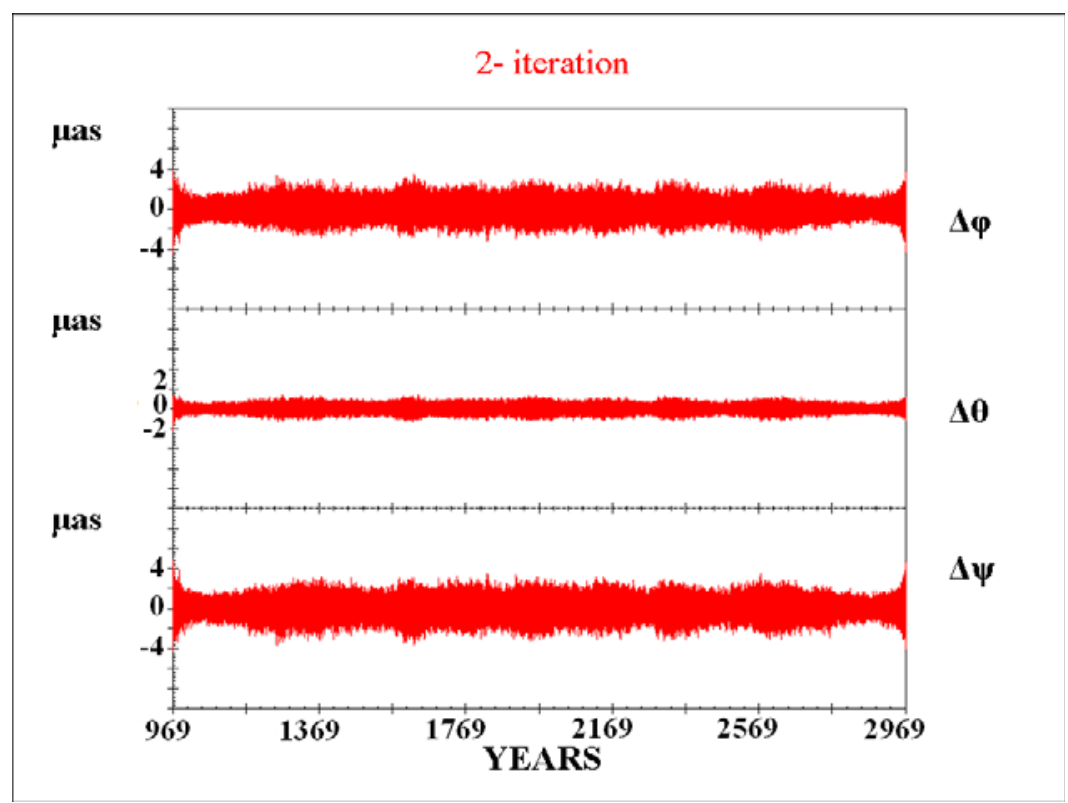

Fig. 7. Discrepancies between the numerical and RERS2012B-2 semi-analytical solutions of the Earth rotation (for the DE406/LE406 ephemeris) over 2000 years after formal removal of the secular trend in the proper rotation angle 
In Figure 7 the discrepancies are depicted between the numerical and RERS2012B-2 semi-analytical solutions of the Earth rotation over 2000 years after formal removal of the secular trend in the proper rotation angle. The discrepancies in the Euler angles decrease after the second iteration of the iterative algorithm and are less than $4 \mu$ as over 2000 years. The process of the iterative algorithm is finished at this step.

This investigation is continued and finished at 6000 years time interval. The discrepancies between the numerical and SMART97 semi-analytical solutions of the rigid Earth rotation (for the DE406/LE406 ephemeris) are obtained in the Euler angles over 6000 year time interval with one day spacing (presented in Figure 8). The residuals have the secular trends, which do not exceed 64 arc seconds.

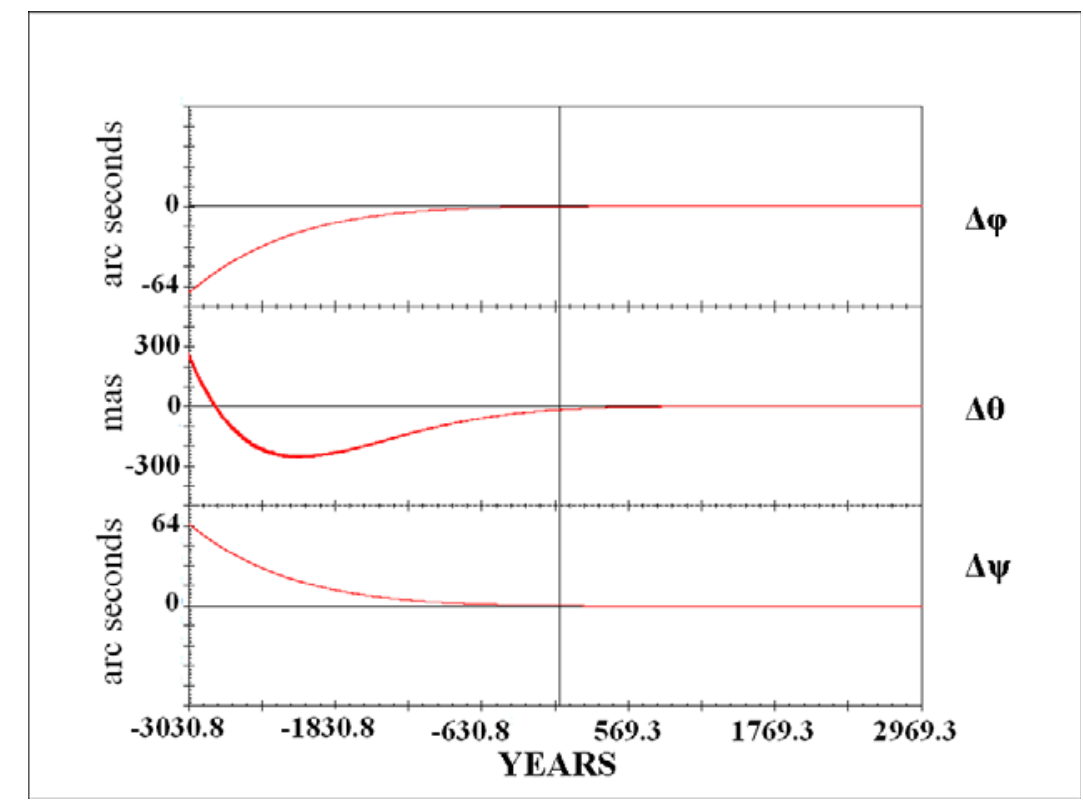

Fig. 8. Discrepancies between the numerical and SMART97 semi-analytical solutions of the Earth rotation (for the DE406/LE406 ephemeris) over 6000 years

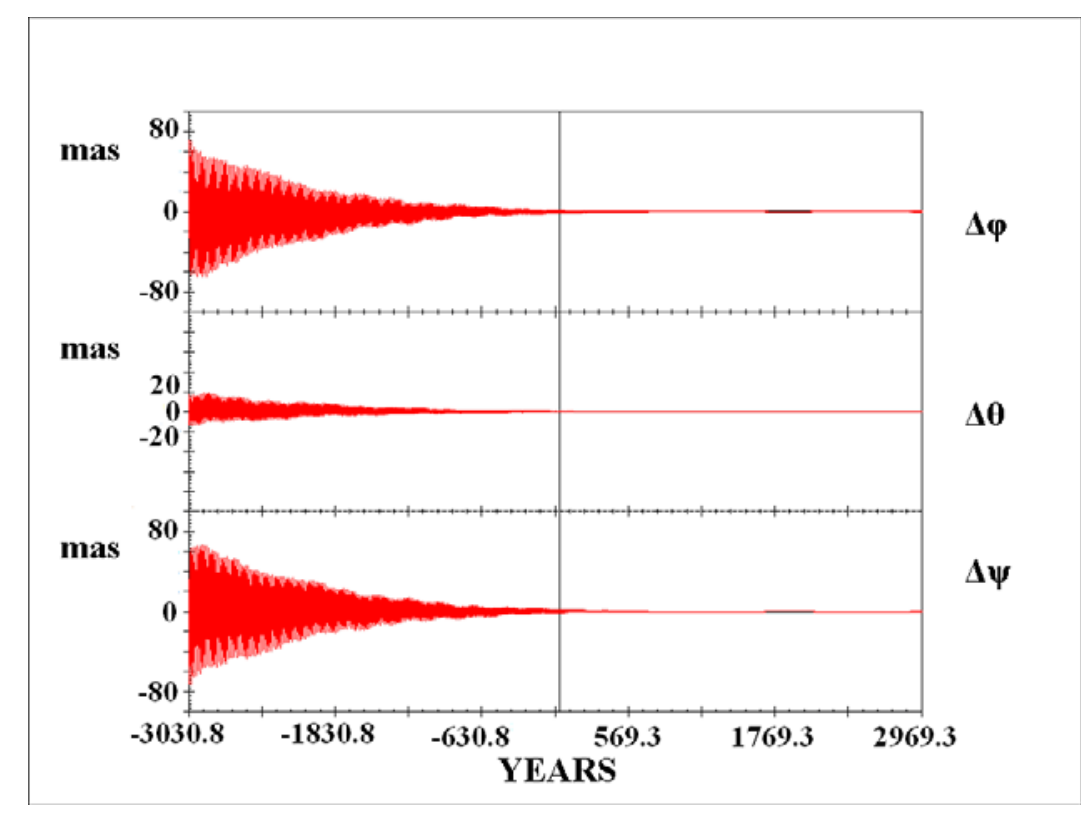

Fig. 9. Discrepancies between the numerical and SMART97 semi-analytical solutions of the Earth rotation (for the DE406/LE406 ephemeris) over 6000 years after formal removal of the secular trends 
In Figure 9 the discrepancies are depicted between the numerical and SMART97 semianalytical solutions of the rigid Earth rotation over 6000 years after formal removal of the secular trends. Here, the residuals do not exceed 80 mas.

In Figure 10 the discrepancies are depicted between the numerical and RERS2012C-1 semi-analytical solutions of the Earth rotation over 6000 years after formal removal of the secular trend in the proper rotation angle. The residuals after the first iteration of the iterative algorithm do not exceed 2 mas.

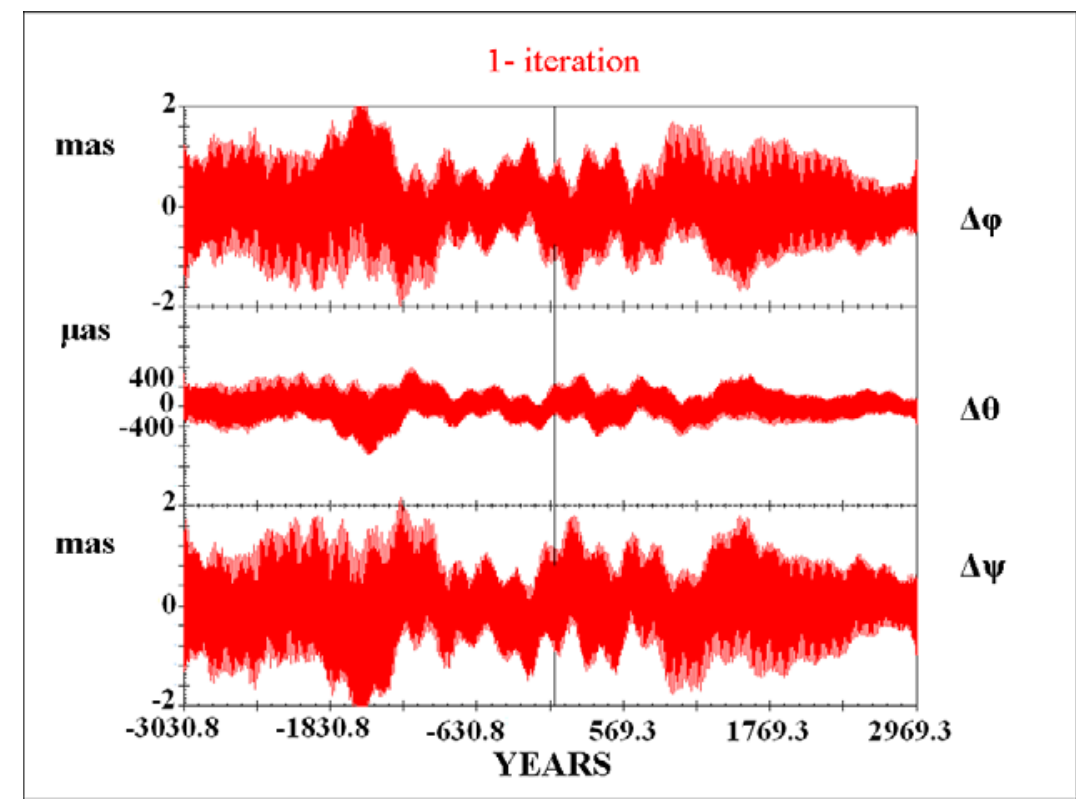

Fig. 10. Discrepancies between the numerical and RERS2012C-1 semi-analytical solutions of the Earth rotation (for the DE406/LE406 ephemeris) over 6000 years after formal removal of the secular trend in the proper rotation angle

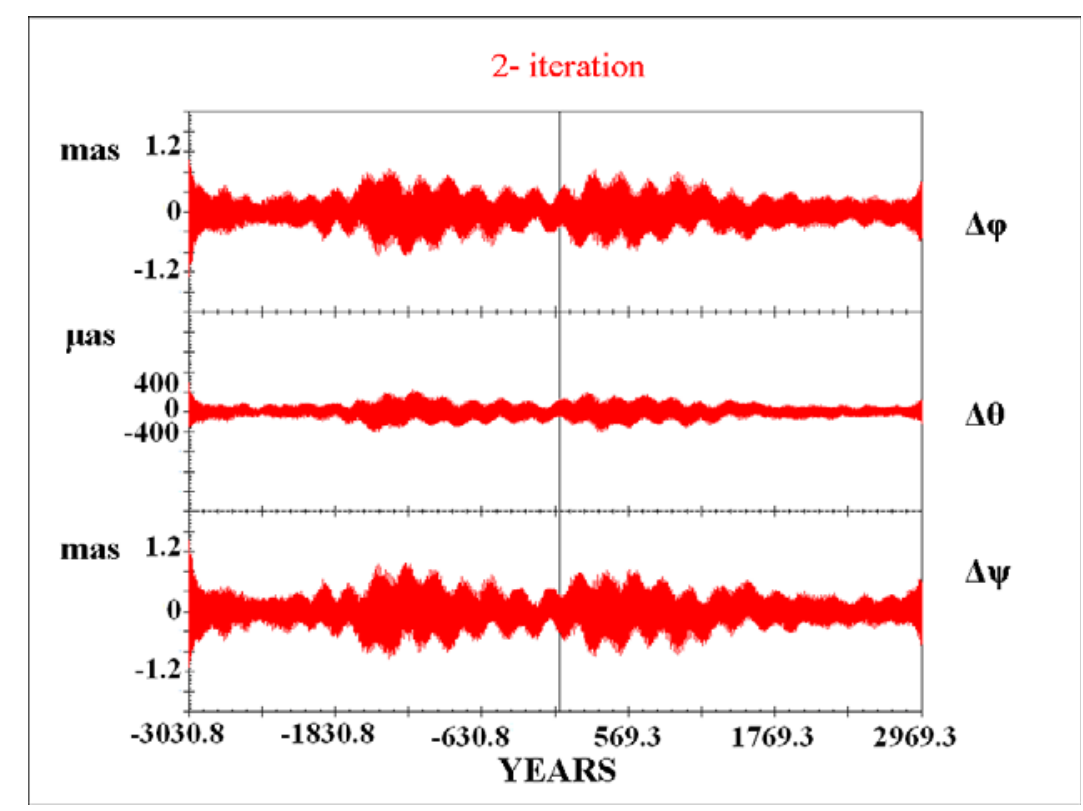

Fig. 11. Discrepancies between the numerical and RERS2012C-2 semi-analytical solutions of the Earth rotation (for the DE406/LE406 ephemeris) over 6000 years after formal removal of the secular trend in the proper rotation angle 
In Figure 11 the discrepancies are depicted between the numerical and RERS2012C-2 semi-analytical solutions of the Earth rotation over 6000 years after formal removal of the secular trend in the proper rotation angle. The residuals after the second iteration of the iterative algorithm do not exceed 1.2 mas.

Figure 12 shows that the residuals between the numerical and RERS2012C-2 (blue color) semi-analytical solutions, after the second iteration, and the residuals between the numerical and RERS2012C-3 (red color) semi-analytical solutions, after the third iteration are very close to each other. Then the process of the iterative algorithm is finished at this step.

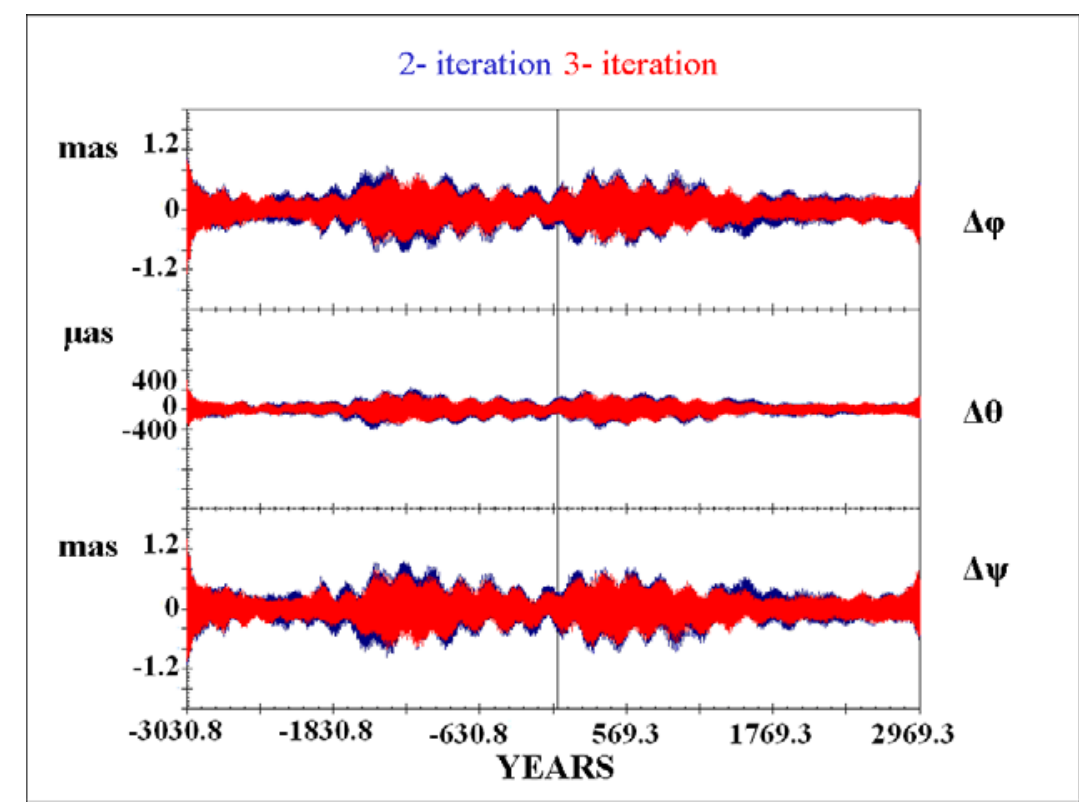

Fig.12. Discrepancies between the numerical and (RERS2012C-2 (blue color) and RERS2012C-3 (red color)) semi-analytical solutions of the Earth rotation (for the DE406/LE406 ephemeris) over 6000 years after formal removal of the secular trend in the proper rotation angle

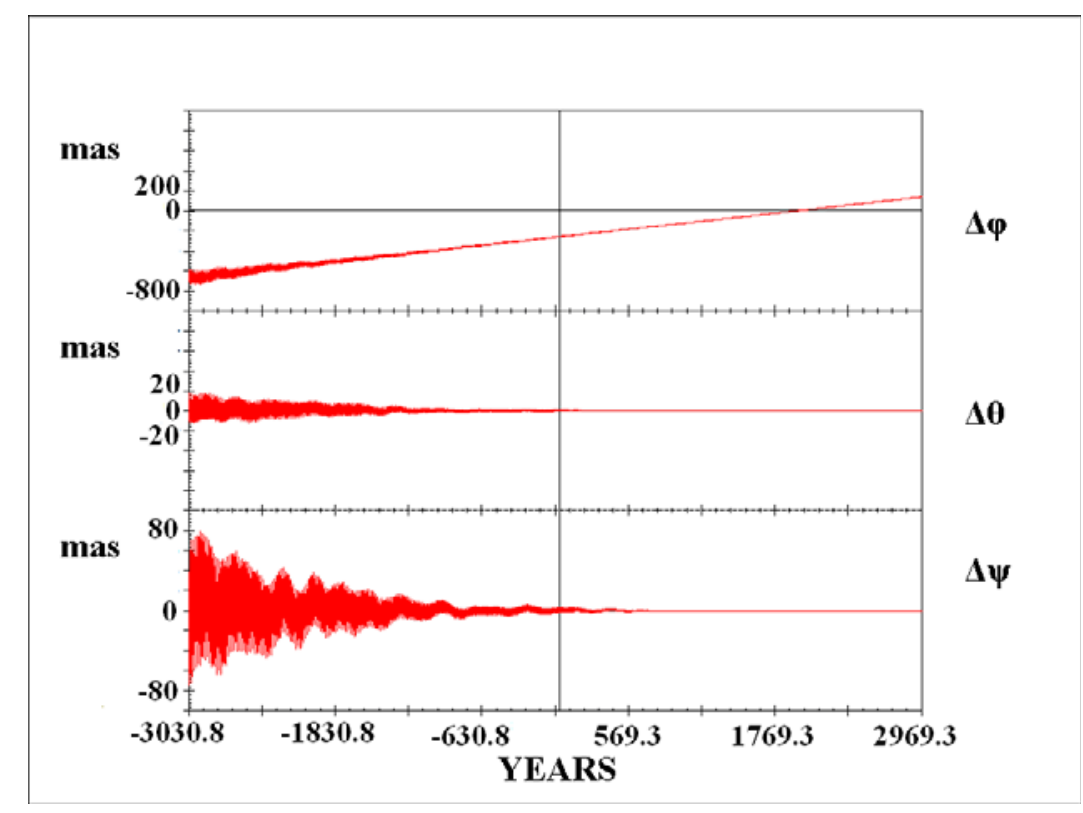

Fig. 13. Discrepancies between the numerical and S9000 semi-analytical solutions of the Earth rotation (for the DE406/LE406 ephemeris) over 6000 years 
The discrepancies between the numerical and S9000 semi-analytical solutions of the rigid Earth rotation (for the DE406/LE406 ephemeris) are obtained in the Euler angles over 6000 year time interval with one day spacing (presented in Figure 13). Only in the proper rotation angle these residuals have the secular trend, which do not exceed 800 mas. Thus, the residuals between the numerical and S9000 semi-analytical solutions (presented in Figure 13) are better than the residuals between the numerical and SMART97 semi-analytical solutions (presented in Figure 8). The appearance of the secular trends in the discrepancies (presented in Figures 8,13 ) due to different ephemeris, which used for the construction of the numerical and semianalytical solutions. Namely, the semi-analytical solution SMART97 was construct with using DE403/LE403 ephemeris, the semi-analytical solution S9000 is dynamically adequate to DE404/LE404 ephemeris and the numerical solutions of this investigation using DE406/LE406 ephemeris.

In Figure 14 the discrepancies are depicted between the numerical and S9000 semianalytical solutions of the rigid Earth rotation over 6000 years after formal removal of the secular trend in the proper rotation angle. Here, the residuals do not exceed 80 mas.

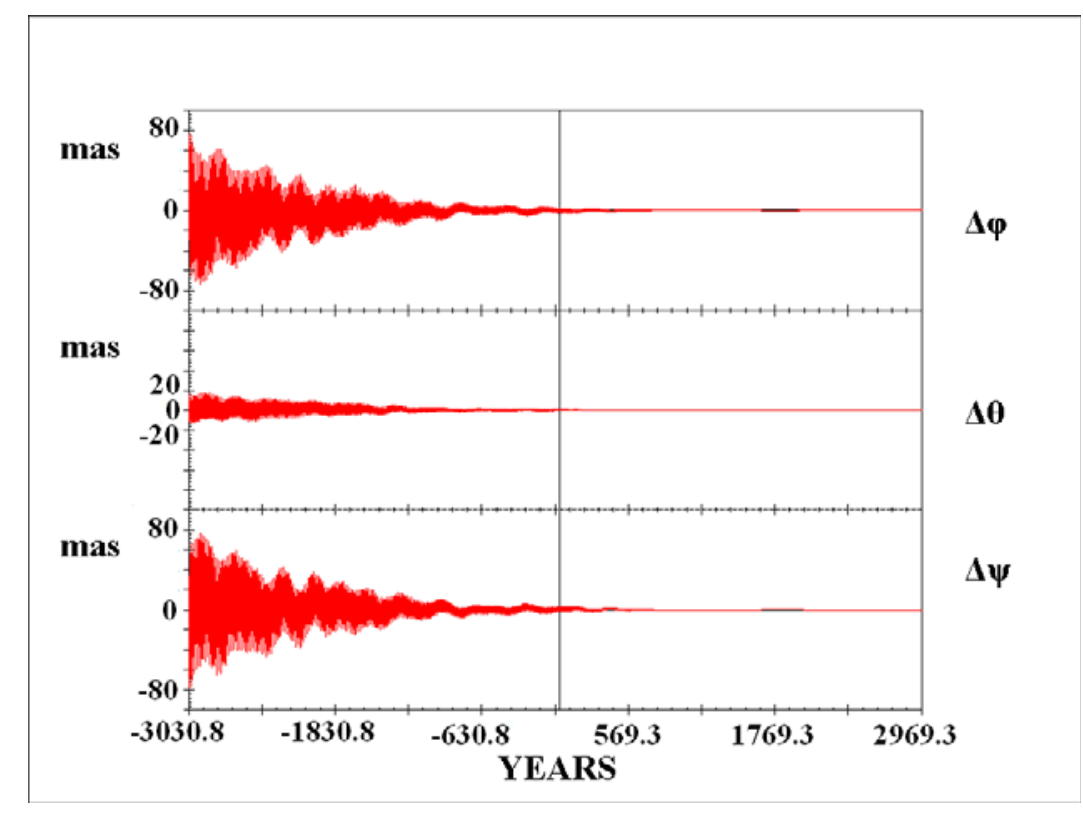

Fig. 14. Discrepancies between the numerical and S9000 semi-analytical solutions of the Earth rotation (for the DE406/LE406 ephemeris) over 6000 years after formal removal of the secular trend in the proper rotation angle

The large values of Poisson terms in the discrepancies at beginning of time interval and small at the end of time interval (presented in Figures 9, 14) are due to uneven ephemeris accuracy over the entire interval of existence. Namely, initial epoch of the ephemeris is 1969 year and the ephemeris accuracy decreases towards the ends of the interval of existence.

In Figure 15 the discrepancies are depicted between the numerical and RERS2012D-1 semi-analytical solutions of the Earth rotation over 6000 years after formal removal of the secular trend in the proper rotation angle. The residuals after the first iteration of the iterative algorithm do not exceed 2 mas.

In Figure 16 the discrepancies are depicted between the numerical and RERS2012D-2 semi-analytical solutions of the Earth rotation over 6000 years after formal removal of the secular trend in the proper rotation angle. The residuals after the second iteration of the 
iterative algorithm also do not exceed 2 mas. So, the process of the iterative algorithm is finished at this iteration.

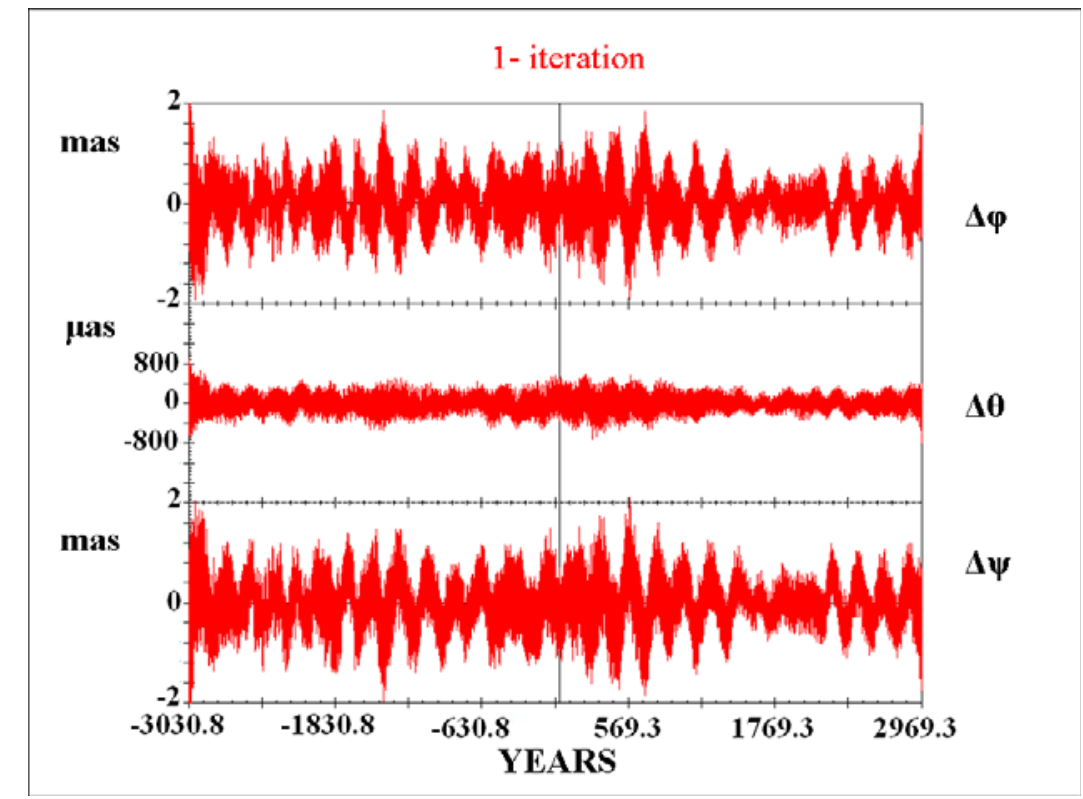

Fig. 15. Discrepancies between the numerical and RERS2012D-1 semi-analytical solutions of the Earth rotation (for the DE406/LE406 ephemeris) over 6000 years after formal removal of the secular trend in the proper rotation angle

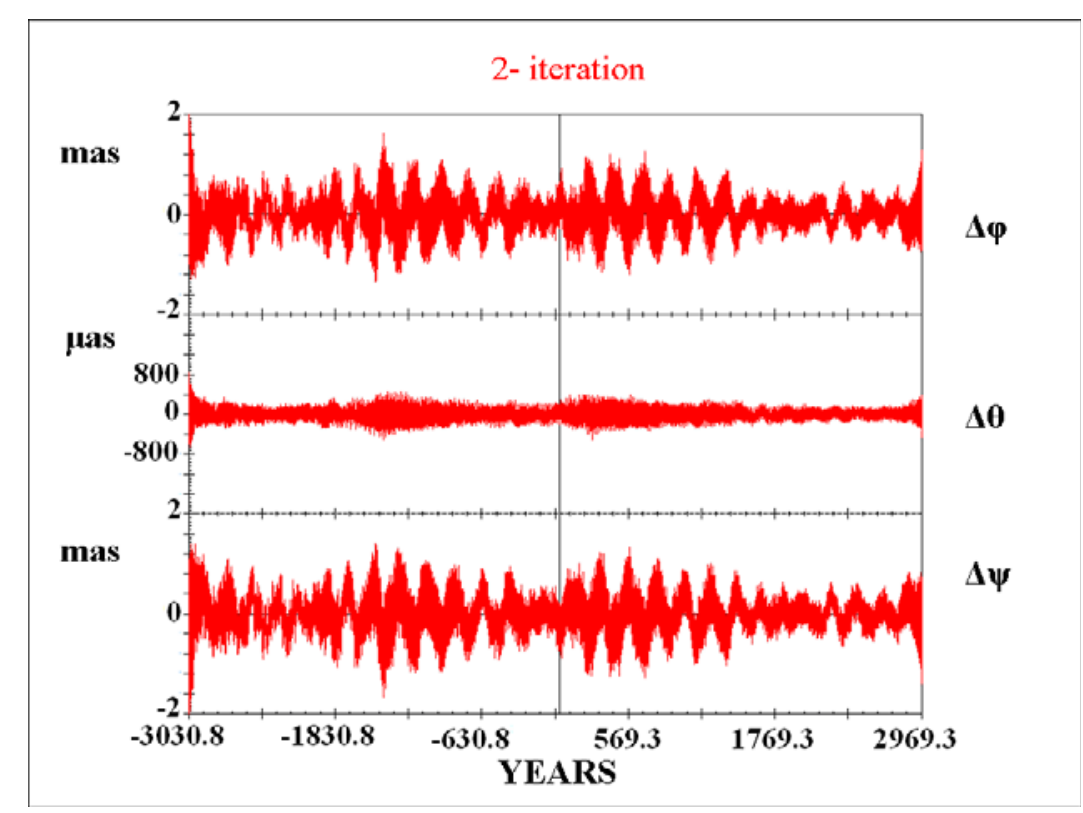

Fig. 16. Discrepancies between the numerical and RERS2012D-2 semi-analytical solutions of the Earth rotation (for the DE406/LE406 ephemeris) over 6000 years after formal removal of the secular trend in the proper rotation angle

\section{CONCLUSIONS}

As the results of this investigation, the new high-precision Rigid Earth Rotation Series RERS2012A/B and RERS2012C/D dynamically adequate to the DE406/LE406 ephemeris over 2000 and 6000 years, respectively, are constructed. 
The new series RERS2012 includes about 4113 periodical and Poisson terms (without including new sub-diurnal and diurnal periodical and Poisson terms). The sub-diurnal and diurnal periodical and Poisson terms have not been investigated in this study. Therefore, they entered into new solutions RERS2012A/C and RERS2012B/D without change from SMART97 and S9000, respectively.

The discrepancies between the numerical solution and RERS2012A/B/C/D do not surpass:

$12 \mu$ as and $4 \mu$ as over 2000 year time interval,

1.2 mas and 2 mas over 6000 year time interval, respectively.

It means a good consistency of RERS2012 series with the DE406/LE406 ephemeris.

\section{ACKNOWLEDGEMENTS}

The authors are very grateful to Prof. Aleksander Brzezinski, Prof. Wieslaw Kosek for very useful discussions, Prof. Janusz Zielinski, Dr. Leszek Jaworski and Dr. Ryszard Zdunek for the grants of financial support.

The investigation was carried out at the Central (Pulkovo) Astronomical Observatory of the Russian Academy of Science and the Space Research Centre of the Polish Academy of Science, under a financial support of the Cooperation between the Polish and Russian Academies of Sciences, Theme No 34. Additional support have been provided by the grants:

EEGS2 (J.B. Zieliński), PECS-EEI (L. Jaworski) and NCN 501-1-95 (R. Zdunek).

\section{REFERENCES}

Eroshkin G.I. (2000): High-Precision method of the Numerical Solution of the Celestial Mechanic problems based on Chebyshev Polynomial Interpolation, In: Book of abstracts, Astrometry, Geodynamics and Celestial Mechanics at the turn of XXIth century, (eds. A.M.Finkelstein et al.) The Conference of the St.Petersburg in the IPA RAS, 19-23 June $2000\}$, pp. 229-230 (in Russian).

Bretagnon P., Francou G., Rocher P., Simon J.L. (1998) SMART97: a new solution for the rotation of the rigid Earth, Astron. Astrophys., Vol. 329, pp. 329-338.

Eroshkin G.I., Pashkevich V.V. and Brzeziński A. (2002): Extension of the high-precision numerical theory of the rigid Earth rotation to the case of a long time interval, Artificial Satellites, 37, 4, pp. 169-183.

Pashkevich V. V., Eroshkin G. I. (2005) Application of the spectral analysis for the mathematical modelling of the rigid Earth rotation, Artificial Satellites, Vol. 40, No. 4, pp. 251-259.

Standish E. M. (1998) JPL Planetary and Lunar Ephemerides, DE405/LE405, JPL Interoffice Memorandum 312.F-98-048.

Received: 2012-11-07,

Reviewed: 2013-01-14,

Accepted: 2013-01-18. 